\title{
Colecistectomía segura: ¿Qué es y cómo hacerla? ¿Cómo lo hacemos nosotros?
}

\author{
Safe cholecystectomy: What is it and how to do it? How do we do it?
}

\begin{abstract}
Alfredo Chama-Naranjo', Jorge Farell R², Víctor José Cuevas $\mathrm{O}^{3}$
1 Médico, residente de Cirugía General, Universidad Nacional Autónoma de México; Hospital Central Sur de Alta Especialidad, Petróleos Mexicanos, Ciudad de México, México.

2 Médico, especialista en Cirugía General, encargado del departamento de Cirugía General; profesor titular, Cirugía General, Universidad Nacional Autónoma de México; Hospital Central Sur de Alta Especialidad, Petróleos Mexicanos, Ciudad de México, México.

3 Médico especialista en Cirugía General, adscrito del departamento de Cirugía General, Hospital Central Sur de Alta Especialidad, Petróleos Mexicanos, Ciudad de México, México.
\end{abstract}

\section{Resumen}

La colecistectomía laparoscópica es uno de los procedimientos más realizados a nivel mundial. La técnica laparoscópica se considera el estándar de oro para la resolución de la patología de la vesícula biliar secundaria a litiasis, y aunque es un procedimiento seguro, no se encuentra exenta de complicaciones. La complicación más grave es la lesión de la vía biliar, que, aunque es poco frecuente, con una incidencia de 0,2 a $0,4 \%$, conduce a una disminución en la calidad de vida y contribuye a un aumento en la morbi-mortalidad. El objetivo de este artículo es reportar nuestra técnica quirúrgica, enfatizando los principios del programa de cultura para una colecistectomía segura, propuesta y descrita por the Society of American Gastrointestinal and Endoscopic Surgeons (SAGES), para minimizar los riesgos y obtener un resultado quirúrgico satisfactorio.

Palabras clave: colecistectomía laparoscópica; procedimientos quirúrgicos mínimamente invasivos; conducto colédoco; visión crítica de seguridad; seguridad del paciente; complicaciones intraoperatorias.

\begin{abstract}
Laparoscopic cholecystectomy is one of the most performed procedures worldwide. The laparoscopic technique is considered the gold standard for the resolution of gallbladder pathology secondary to lithiasis, and although it is a safe procedure, it is not without complications. The most serious complication is the injury to the bile duct, which, although rare, with an incidence of $0.2 \%$ to $0.4 \%$, leads to a decrease in quality of life and contributes to an increase in morbidity and mortality. The objective of this article is to report our surgical technique, emphasi-
\end{abstract}

Fecha de recibido: 24/06/2020 - Fecha de aceptación: 13/09/2020 - Fecha de publicación en línea: 11/02/2021

Correspondencia: Alfredo Chama Naranjo, Anillo Periférico 4091, Fuentes del Pedregal, Tlalpan, 14140, Ciudad de México, México. Teléfono: +52 5581 007189. Correo electrónico: alfredchamaa@gmail.com

Citar como: Chama-Naranjo A, Farell RJ, Cuevas VJ. Colecistectomía segura: ¿Qué es y cómo hacerla? ¿Cómo lo hacemos nosotros? Rev Colomb Cir. 2021;36:324-33. https://doi.org/10.30944/20117582.733

Este es un artículo de acceso abierto bajo una Licencia Creative Commons - BY-NC-ND https://creativecommons.org/licenses/by-ncnd/4.0/deed.es 
zing the principles of the program for a safe cholecystectomy, proposed and described by the Society of American Gastrointestinal and Endoscopic Surgeons (SAGES), to minimize the risks and obtain a satisfactory surgical result.

Keywords: laparoscopic cholecystectomy; minimally invasive surgical procedures; common bile duct; critical view of safety; patient safety; intraoperative complications.

\section{Introducción}

La enfermedad por cálculos biliares es muy frecuente a nivel mundial. Afecta a la población del continente americano con una prevalencia que oscila entre un 11 y un $35 \%$; en Estados Unidos se ha descrito una prevalencia de 10 a $15 \%$, con una frecuencia de 750.000 colecistectomías al año ${ }^{1}$, mientras México presenta una prevalencia de 14,3 $\%$, una distribución más alta que en otros países desarrollados ${ }^{2}$. Es una de las principales causas de consulta en cirugía general y se considera la enfermedad quirúrgica más prevalente en México, con 69 mil intervenciones al año $0^{3,4}$.

Desde la introducción de la colecistectomía laparoscópica (CL) por Lagenbuch en los años 80 's, la CL ofrece ventajas en comparación con la colecistectomía abierta, debido a que presenta una mejor visibilidad quirúrgica, disminución del dolor postquirúrgico y mejor control con analgésicos de uso común, lo que se traduce en una menor estancia hospitalaria y una recuperación más temprana por parte del paciente, además de un mejor resultado estético, por lo que fue considerada como el estándar de oro en el consenso de Bethesda en $1992^{5,6}$.

Aunque la CL se considera un procedimiento seguro, no está exenta de complicaciones, de las cuales las más frecuentes son sangrado, seroma, biloma, fuga biliar e infección del sitio operatorio, que causan un $7 \%$ de morbilidad, con una tasa de mortalidad de $1,2 \%{ }^{7}$. La complicación más compleja es la lesión de la vía biliar (LVB), con una incidencia de 0,2 a $0,4 \%{ }^{8}$, que generalmente se debe a una identificación errónea de las estructuras anatómicas principales y se asocia con una serie de eventos que disminuyen la calidad de vida de los pacientes, con la necesidad de intervenciones y procedimientos adicionales, que contribuyen al aumento en la mortalidad y costos en salud ${ }^{9}{ }^{\mathrm{1} 10}$.
Se han propuesto varios métodos para realizar una colecistectomía segura. La visión crítica de seguridad, adoptada por Steven Strasberg desde 1995, ha sido una herramienta útil para la correcta identificación de las estructuras anatómicas ${ }^{11}$. The Society of Gastrointestinal and Endoscopic Surgeons (SAGES) en el año de 2014, con el objetivo de mejorar la seguridad de la CL y reducir la tasa de lesiones biliares, formó la cultura de colecistectomía segura en torno a este procedimiento ${ }^{12}$. El principal objetivo de este artículo es transmitir cómo el servicio quirúrgico en nuestro hospital practica la colecistectomía laparoscópica segura.

\section{Preparación preoperatoria}

El American Collage of Surgeons (ACS) promueve un programa de calidad para cirugías electivas, en hospitales y clínicas, para optimizar la salud de los pacientes previo a la cirugía. STRONG for Surgery es un programa que permite integrar una lista de verificación en la fase preoperatoria para identificar factores de riesgo que pueden conducir a complicaciones postoperatorias. El propósito es proporcionar intervenciones apropiadas para garantizar un resultado exitoso posterior a la cirugía. La lista de verificación se integra por 8 áreas que son determinantes para los resultados de la cirugía ${ }^{13}$ :

1) Nutrición,

2) Control glicémico,

3) Manejo de medicamentos,

4) Suspensión del tabaco,

5) Manejo seguro y efectivo del dolor postquirúrgico,

6) Medidas anti-delirium,

7) Rehabilitación,

8) Educación para el paciente. 
En nuestra institución los pacientes se presentan en consulta externa para valoración preoperatoria y programación quirúrgica, con estudios de biometría hemática, tiempos de coagulación, química sanguínea con pruebas de función hepática y ecografía hepatobiliar. Se evalúa el riesgo de coledocolitiasis según los criterios de ASGE ${ }^{14}$. Recomendamos el uso de antibiótico profiláctico para prevenir infecciones postoperatorias, además del uso de medidas antitromboembólicas ${ }^{15,16}$. La inserción de sonda nasogástrica intraoperatoria no se recomienda de rutina, excepto en los casos de distensión gástrica, para mejor la identificación del campo quirúrgico ${ }^{17}$. La sonda Foley no se usa de forma rutinaria. ${ }^{18} \mathrm{Si}$ alguna de las sondas es utilizada durante el preoperatorio, se recomienda su retiro antes del término de la cirugía, debido a que favorece una recuperación más rápida para el paciente.

En el escenario de una colecistectomía difícil, previo a la cirugía el cirujano debe considerar diversas opciones para optimizar el resultado. Se conocen varios factores predictores de colecistectomía difícil, como son género masculino, edad mayor a 70 años, obesidad, antecedente de diabetes mellitus, colecistitis aguda, cirugía abdominal previa y el factor cirujano. Otros predictores menos importantes son historia prolongada de enfermedad vesicular (más de un año del diagnóstico), leucocitosis y signos sistémicos de sepsis. Con la ayuda de la ecografía, otros factores predictores son engrosamiento de la pared vesicular (más de $4 \mathrm{~mm}$ ), líquido perivesicular, vesícula calcificada o escleroatrófica, cálculos grandes o impactados en la bolsa de Hartmann y dilatación de la vía biliar. Otras situaciones que pueden condicionar a una colecistectomía difícil son un hígado grande, alteraciones anatómicas, cirrosis hepática, presencia de fístulas colecisto-intestinales y cáncer de vesícula biliar ${ }^{19}$.

\section{Técnica quirúrgica}

\section{Acceso abdominal y colocación de puertos}

El paciente se coloca en posición supina con el brazo izquierdo plegado, para facilitar la colan- giografía transoperatoria (CTO) en caso de ser necesario durante el procedimiento. El cirujano se ubica a la izquierda del paciente y el primer asistente a la derecha, para acceder a la cavidad abdominal y realizar el neumoperitoneo (figura 1). El acceso al abdomen para colocar el primer puerto de trabajo de $10 \mathrm{~mm}$ (T1) puede lograrse por medio de una técnica abierta o cerrada. En pacientes que presentan cicatrices sobre la línea media o periumbilicales se debe optar por un acceso alternativo, con una aguja de Veress en el cuadrante superior izquierdo (punto de Palmer) o mediante una incisión epigástrica abierta ${ }^{20}$. En los pacientes con obesidad, se puede obtener acceso por técnica cerrada con aguja de Veress, justo a la derecha de la línea media, $15 \mathrm{~cm}$ por debajo de la apófisis xifoides, debido a que el ombligo es desplazado hacia abajo en estos pacientes, y se debe de optar por el acceso más familiarizado por parte del cirujano.

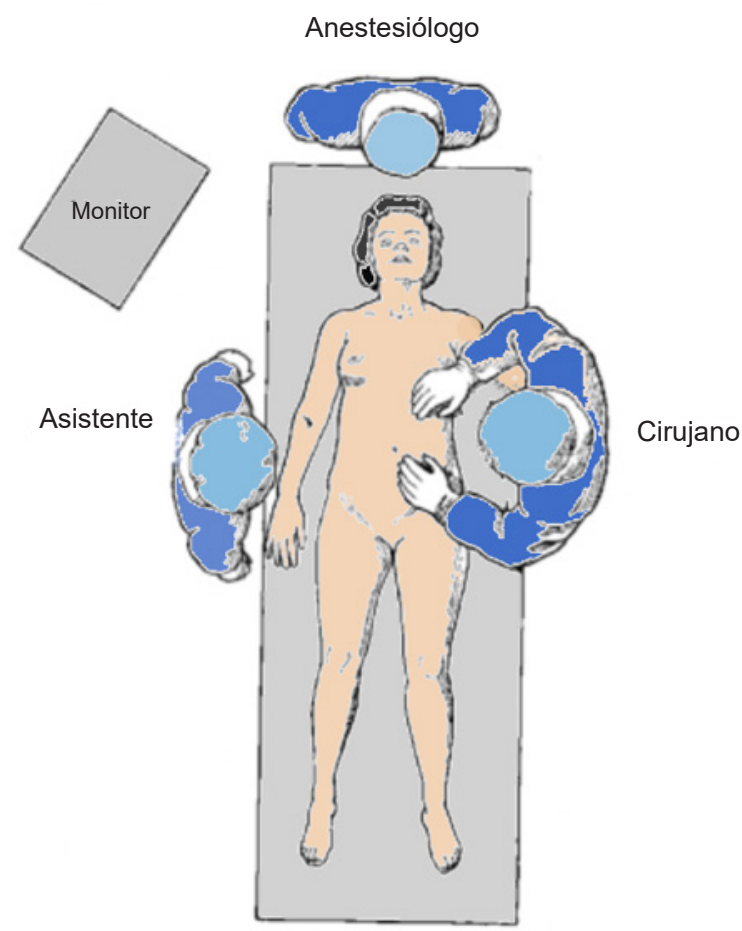

Figura 1. Posición del paciente para colecistectomía laparoscópica. 
Después de la creación del neumoperitoneo y la laparoscopía diagnóstica, se procede a colocar el segundo puerto de trabajo de $10 \mathrm{~mm}$ (T2), 2 $\mathrm{cm}$ por debajo de la apófisis xifoides y en el borde derecho del ligamento falciforme. Dos puertos de trabajo de $5 \mathrm{~mm}$ son colocados en la línea clavicular media subcostal derecha (T3) y la posición subcostal lateral derecha (T4). Previo a la colocación de los trocares, sugerimos la infiltración con ropivacaína al 0,75\%, 5 mililitros en cada puerto de trabajo, para disminuir el dolor postoperatorio $^{21}$. Los trócares de trabajo que utiliza el cirujano son T2 y T3 (figura 2).

\section{Procedimiento:}

Paso 1. Disección del triángulo hepatocístico.

Una vez que se han reconocido las estructuras anatómicas, la vesícula biliar (VB) se eleva y retrae sobre el hígado, mediante tracción del fondo en sentido cefálico, con una pinza a través del puerto T4. La tracción lateral del cuello o el infundíbulo de la VB se aplica con la ayuda de otra pinza, a

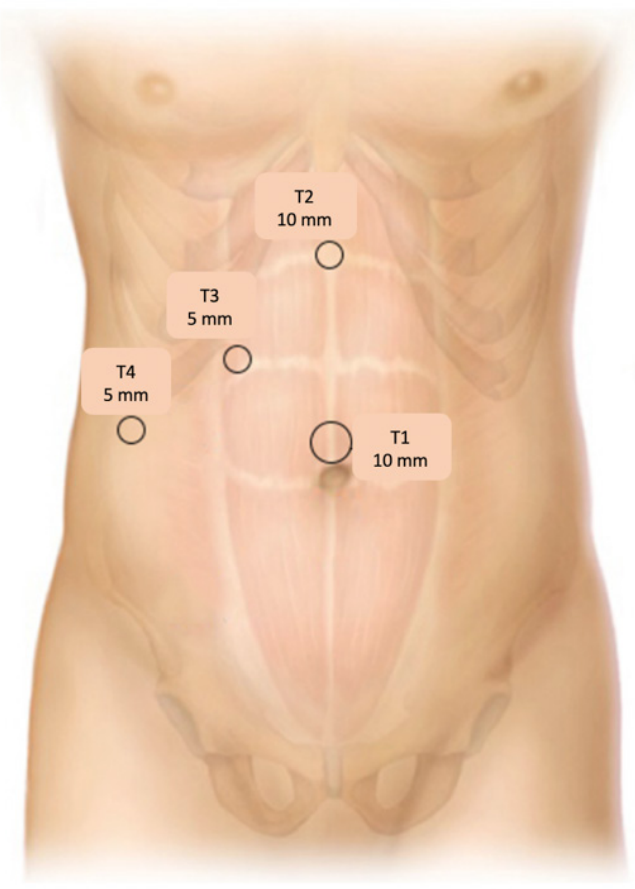

Figura 2. Posición de los trocares para colecistectomía laparoscópica través del puerto T3. El ayudante quirúrgico generalmente mantiene una tensión constante en T4.

Recomendamos como primer paso la búsqueda del surco de Rouviere, ya sea en forma de cicatriz, hendidura o surco, para iniciar la disección por arriba de este surco, evitando cualquier disección por debajo de este punto de referencia, para eliminar cualquier peligro de LVB $^{22}$ (figura 3). La mano del cirujano, a través de T3, puede manipular el cuello de la VB para exponer la cara anterior (medial) y posterior (lateral), según sea necesario. Si la VB se encuentra a tensión, se recomienda descomprimirla con un dispositivo de aspiración con aguja, para evitar perforaciones con derrames de bilis y cálculos biliares. Si hay adherencias, se eliminan con tracción o con corte frío, evitando el uso de energía cerca del duodeno y de la vía biliar principal.

La disección comienza incidiendo el peritoneo visceral, a lo largo del borde de la VB en ambos lados (anterior y posterior), para abrir el peritoneo que recubre el triángulo hepatocístico. Esto debe llevarse hacia arriba a lo largo de la VB, y hacia el fondo vesicular, en su interfaz con el hígado. Se necesita una combinación de disección roma y uso cauteloso de electrocauterio (20 W), con el objetivo de limpiar el triángulo de tejido graso y fibroso, a consideración del cirujano.

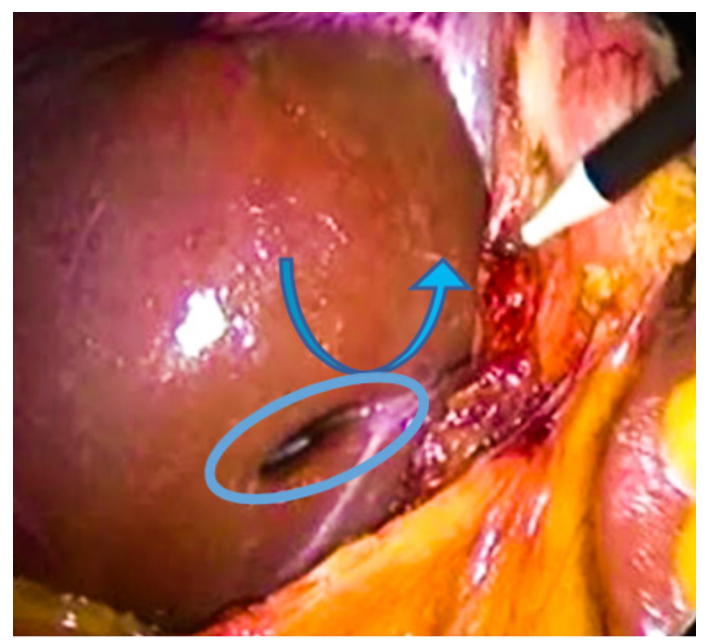

Figura 3. Surco de Rouviere. Punto de referencia para iniciar la disección en la colecistectomía laparoscópica. 
Paso 2. Visión crítica de seguridad.

La visión crítica de seguridad requiere que se cumplan tres criterios:

1) El triángulo hepatocístico (formado por el conducto cístico, el conducto hepático común y el borde inferior del hígado) debe estar limpio de todo el tejido graso y fibroso. El conducto biliar común y el conducto hepático común se buscan, pero no se exponen para su disección.

2) El tercio inferior de la VB se separa hasta en un $30 \%$ del hígado, para exponer la placa cística. La placa cística se define como el lecho hepático adherido a la VB y representa la fosa de la VB.

3) Deben observarse dos, y solo dos estructuras que ingresen a la VB, que representan el conducto y la arteria císticos, en una vista anterior y posterior ${ }^{9}$. Una vez establecida esta visión, recomendamos una pausa y una confirmación entre el cirujano y el ayudante quirúrgico, antes de cortar cualquier estructura (figura 4). En esta pausa, la identificación de la anatomía aberrante es crítica, una com-

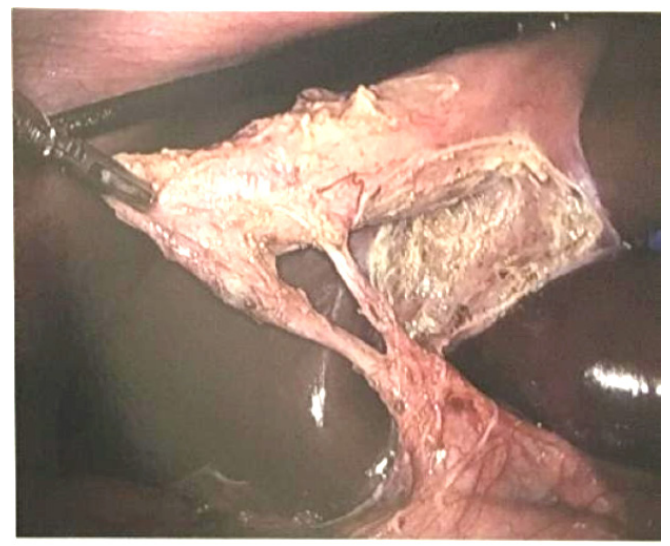

Figura 4. Visión crítica de seguridad. 1) El triángulo hepatocístico se ha diseccionado sin grasa ni tejido fibroso, no se muestra el conducto biliar principal. 2) La base de la vesícula biliar se ha diseccionado en su tercio inferior exponiendo la placa cística. 3) Dos y solo dos estructuras ingresan a la vesícula biliar y pueden verse circunferencialmente. prensión profunda y una apreciación de la anatomía aberrante es importante para minimizar el riesgo de una LVB. Una consideración común es asegurar que la arteria hepática derecha no se confunda con la arteria cística o la rama accesoria posterior en el área de la placa cística. En este punto se puede considerar el uso de CTO, en caso de ser necesario.

Paso 3. La arteria cística se secciona y se divide.

En nuestra institución, para minimizar los costos, para la arteria cística se utiliza una engrapadora reutilizable con grapas de $8 \mathrm{~mm}$, utilizando dos grapas en el lado proximal y una grapa en el lado distal (pieza quirúrgica), con un espacio que permita el corte entre ambos. Las tijeras laparoscópicas se usan para cortar la arteria. Se debe tener cuidado durante este proceso para no desplazar las grapas proximales. Nuestro equipo quirúrgico deja una pequeña banda de tejido más allá del borde de las grapas, para evitar el desplazamiento accidental durante la manipulación del conducto cístico.

Si se planea la CTO, se coloca una grapa en el cuello de la VB, en el extremo superior de la unión del conducto cístico con la VB; esta división de la grapa puede garantizar que la muestra distal se desaloje.

Paso 4. Colangiografía transoperatoria (CTO) y división del conducto cístico.

Es nuestra práctica quirúrgica no realizamos de forma rutinaria la CTO. Generalmente, la CTO se realiza de forma selectiva y las indicaciones son la sospecha de cálculos en la vía biliar (por alteración de las pruebas de función hepática o antecedente de pancreatitis de origen biliar), un colédoco o conducto biliar común dilatado (más de $6 \mathrm{~mm}$ ), la duda en la anatomía o preocupación por lesión de la vía biliar, y el antecedente de baipás gástrico en Y de Roux, que impide la colangiopancreatografía retrógrada endoscópica (CPRE) posterior ${ }^{23}$.

Para realizar una CTO se coloca una sola grapa en la unión del conducto cístico y el infundíbulo de la VB. El conducto cístico se incide parcialmente con tijera laparoscópica y se procede a dilatar las válvulas espirales de Heister del conducto cístico 
con el disector laparoscópico. Luego se inserta una sonda 4 French en el conducto cístico, usando una pinza con clamp para asegurar el catéter. Para realizar la CTO, las capturas de imagen se hacen en un solo punto, ajustando la posición del arco en C de modo que el árbol biliar y el duodeno se puedan visualizar en el centro del marco. Nosotros utilizamos dos jeringas de $20 \mathrm{ml}$, una contiene solución salina y la otra una mezcla 50-50 de solución salina y medio de contraste yodado, conectados a través de una llave de tres vías para aplicarlo.

Inicialmente se aplica solución salina en el conducto cístico y luego la mezcla con medio de contraste, para visualizar con fluoroscopia. Se debe observar el llenado retrógrado del conducto hepático común, así como de las ramas derecha e izquierda en el hígado, y el contraste debe fluir libremente hacia el duodeno, asegurando una vía biliar sin defecto de llenado. Si se encuentra dificultad con el llenado retrógrado del conducto, se debe colocar al paciente en Trendelenburg y comprimir suavemente el conducto distal con una pinza. Si se visualiza un cálculo en el conducto biliar, se debe tomar la decisión de intentar una exploración transcística del conducto biliar común, una coledocotomía laparoscópica o una derivación para después llevar a CPRE postoperatoria, de acuerdo con la experiencia y preferencia del cirujano y con los recursos disponibles de la institución. Una vez que se obtiene una colangiografía libre de defectos de llenado, se retira la sonda, se colocan las grapas, se corta y divide el conducto cístico.

Si no se realiza CTO, en el conducto cístico se aplican tres grapas, dos en el lado proximal y una en el lado de la pieza quirúrgica, y con la ayuda de la tijera laparoscópica se realiza la división de la estructura. Cuando el conducto cístico se encuentre dilatado o engrosado, recomendamos usar una sutura absorbible (2-0), para realizar un nudo extracorpóreo, o utilizar un endoloop, para asegurar el conducto en el lado proximal.

Paso 5. Separación de la vesícula biliar del lecho hepático.

Después de dividir el conducto cístico, se realiza una disección retrógrada de la VB del lecho hepáti- co. En nuestro centro, generalmente utilizamos un dispositivo de energía monopolar con un gancho en forma de L (hook) para disecar la vesícula biliar del hígado. Debe de mantenerse en el plano entre la vesícula biliar y el lecho hepático para evitar la perforación de la VB y riego de bilis o litos en la cavidad abdominal. El derrame de bilis y cálculos no debería tener consecuencias adversas, siempre que se eliminen del campo. La entrada al lecho hepático puede provocar sangrado o fuga de bilis desde un conducto superficial subhepático. La adecuada manipulación de la VB, para mantener una tracción moviendo el cuello de la VB hacia adelante y hacia atrás, optimiza la visualización y ayuda a mantener la tensión en la línea de disección, lo que facilita este paso en la cirugía.

En el contexto de una VB difícil por colecistitis aguda, el uso de un dispositivo de energía avanzado, como un coagulador ultrasónico, ayuda a mantener una mejor hemostasia y producir menos humo, lo que hace que la disección sea más fácil y eficiente. Después de dividir la VB de la placa, se debe de realizar la hemostasia necesaria para prevenir riesgo de sangrado. El lecho hepático se irriga y cualquier componente de sangre, bilis o litos se deben de retirar. En caso de colecistitis aguda, la VB se coloca dentro de una bolsa de pieza quirúrgica y se retira por el puerto de $10 \mathrm{~mm}$, para disminuir el riesgo de infección del sitio operatorio. En casos selectivos, la bolsa de pieza quirúrgica puede no ser necesaria, y no hay evidencia que justifique su uso de forma rutinaria ${ }^{24}$.

Paso 6. Extracción de pieza quirúrgica y retiro de puertos.

En nuestra institución, con fines de disminuir costos de material, se puede utilizar un guante estéril número 8 confeccionado en el extremo distal con una sutura anudada para la extracción de la pieza quirúrgica (figura 5). Una vez que la pieza quirúrgica está en la bolsa, se debe extraer por el puerto de $10 \mathrm{~mm}$, ya sea en el ombligo o en la región epigástrica (T1 o T2). En algunas ocasiones es necesario extender la incisión de la piel y la apertura de la fascia, especialmente si hay múltiples cálculos, un cálculo grande o una VB engrosada. Después 
de retirar la muestra, se verifica hemostasia del lecho quirúrgico y se considera la necesidad de colocar un drenaje, solo ante sospecha de fuga biliar. Los puertos se ventilan para eliminar el gas $\mathrm{CO}_{2}$ residual. La fascia en el sitio de los puertos de $10 \mathrm{~mm}$ debe cerrarse con una sutura absorbible (0) o una sutura similar, para prevenir una hernia postincisional $^{25}$. La piel se cierra con una sutura subcuticular de monofilamento absorbible.

\section{Postoperatorio}

Durante este periodo generalmente se maneja al paciente con analgésicos intravenosos a demanda del paciente y se recomienda continuar con Paracetamol o antiinflamatorio no esteroideo (AINE) ${ }^{21}$. Consideramos la escala simplificada de Apfel para administrar antieméticos ${ }^{26}$. Se reinicia la vía oral tan rápido como sea tolerada por el paciente y la estancia hospitalaria generalmente es de 2,4 días ${ }^{27}$. La administración de analgésicos se continua vía oral durante 3 a 4 días posterior a la cirugía ${ }^{28}$. Se hace el seguimiento por consulta externa en 10 días para valorar la evolución del postquirúrgico y posible alta médica.

\section{Colecistectomía segura}

La CL es el estándar de oro para la patología de vesícula biliar secundario a litiasis, debido a los

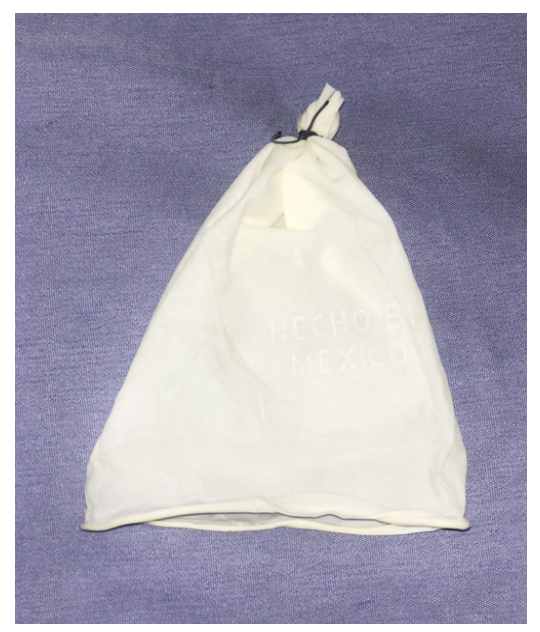

Figura 5. Guante estéril número 8, confeccionado para la extracción de la pieza quirúrgica. beneficios que presentan los pacientes ${ }^{29}$. Sin embargo, desde la introducción de la laparoscopia, la LVB se incrementó, ocurriendo en 3 de cada 1000 procedimientos $^{30}$. Desde 1990, SAGES ha aplicado pautas para realizar una colecistectomía segura, que se actualizan periódicamente. En el consenso de Delphi en 2015, SAGES publicó las siguientes estrategias como parte del programa de cultura para colecistectomía segura y minimizar el riesgo de LVB $^{31}$ :

1. Establecer la identificación de la visión crítica de seguridad: La VCS puede ser comprobada usando una doble visión, anterior y posterior, de las estructuras (figura 6).

2. Comprender la anatomía aberrante potencial en todos los casos.

3. Tener presente el uso de colangiografía o cualquier otro método de imagen para valorar la vía biliar en el transoperatorio. Los estudios han demostrado que el uso de CTO disminuye la incidencia de $\mathrm{LVB}^{32}$.

4. Realizar un "tiempo de espera" durante el procedimiento, previo a cortar o seccionar cualquier estructura ductal, para confirmar que se ha logrado una VCS con doble visión y descartado anatomía aberrante.

5. Reconocer cuando la disección se encuentra en una zona de riesgo y terminar el procedimiento con un método seguro: El criterio del cirujano es indispensable cuando no se obtiene una exposición adecuada de la VCS,

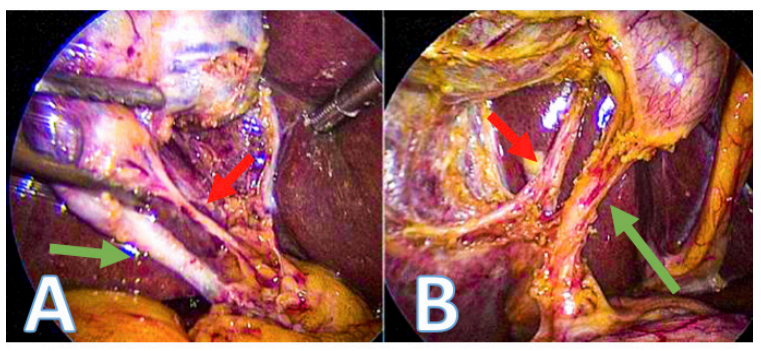

Figura 6. Doble visión para establecer la visión crítica de seguridad. A) Vista anterior B) Vista posterior. Flecha verde: Conducto cístico. Flecha roja: Arteria cística 
se presenta sagrado, extensa fibrosis o inflamación. Se debe considerar la colecistectomía subtotal (CST) o la conversión de la cirugía abierta, basado en la experiencia del cirujano.

6. Tener presente la necesidad de pedir ayuda a otro cirujano en caso de presentar dificultad. El consejo de un segundo cirujano es muy útil cuando las condiciones de la disección se complican, la anatomía no se encuentra clara o en condiciones difíciles consideradas por el cirujano ${ }^{12}$.

\section{Colecistectomía difícil}

El término de colecistectomía difícil hace referencia a la extracción quirúrgica de la vesícula biliar, cuando existen algunas condiciones asociadas del mismo órgano, que no permiten una disección fácil, rápida y cómoda de la vesícula biliar, lo que se traduce en prolongación del tiempo quirúrgico y aumento en el riesgo de complicaciones para el paciente ${ }^{33}$.

Dentro de los elementos que se toman en cuenta para la definición de colecistectomía difícil están utilizar más de dos intentos con aguja de Veress o algún otro método alternativo, como la técnica abierta, disección de adherencias con uso de electrocauterio, necesidad de instrumentos especiales para el manejo de la vesícula biliar, más de 20 minutos de disección para identificar el triángulo de Calot, disección del lecho hepático que requiere más de 20 minutos o perforación de la vesícula biliar. Otras dificultades que definen una colecistectomía difícil incluyen aumentar la incisión inicial de la piel durante la extracción de la vesícula biliar o que ocurra derrame de cálculos o bilis hacia la cavidad abdominal, el tiempo de extracción mayor a 60 minutos desde la inserción de la aguja de Veress y la presencia de variantes anatómicas o la falla en la identificación de estructuras, que requiere algún otro método de salvamento para finalizar la cirugía ${ }^{19}$.

En estas circunstancias, cuando durante una colecistectomía no puede visualizarse el triángulo de Calot, se debe de optar por un procedimiento de salida, de los cuales se han descrito dos vías al- ternas para el término de la cirugía, lo que se ha añadido como "punto de inflexión". Estas son la colecistectomía subtotal (CST) "fenestrada" y "reconstructiva" ${ }^{34}$. En casos especiales, como cuando durante el procedimiento quirúrgico se encuentra fibrosis del cístico, lito impactado en el cístico o sangrado, o cuando es técnicamente insegura, no se puede llevar a cabo la colecistectomía total. Nosotros sugerimos realizar un procedimiento de Estes $^{35}$, el cual consiste en dividir la vesícula biliar desde el fondo hasta la bolsa, a un cm del conducto cístico, ligando este último conducto. Se intenta obtener el efecto de una colecistectomía completa. Se retiran los litos, dejando la vesícula abierta en toda su extensión y una porción de vesícula biliar unida al hígado, y posteriormente se colocan drenajes.

La CST reconstructiva deja un remanente de vesícula biliar, creando una neo-vesícula. Tiene riesgo de fuga biliar y de presentar recurrencia de colelitiasis sintomática. En casos de mayor riesgo de sangrado, como en pacientes con cirrosis o coagulopatías, se recomienda no quitar la pared posterior de la vesícula para disminuir la probabilidad de sangrado y acortar el tiempo quirúrgico ${ }^{36}$.

En la CST fenestrada se cierra el conducto cístico por medio de una sutura absorbible (2-0). Al resecar la mayor parte de vesícula biliar, no suele estar asociada con recurrencia de colelitiasis, sin embargo, presenta mayor riesgo de fuga biliar. $\mathrm{Si}$ después de 7 días persiste la fuga biliar, generalmente se considera necesario el apoyo terapéutico con el servicio de endoscopía, para realizar colangiopancreatografía retrógrada endoscópica (CPRE) para el tratamiento de la complicación. Las fugas biliares con CST se presentan con una incidencia de alrededor de un $20 \%$, mientras en nuestro centro se ha reportado una incidencia de fuga biliar para la CST fenestrada y reconstructiva de un $2,2 \%$.

El riesgo para los pacientes con CST es la necesidad de intervenciones secundarias en casi el $40 \%$ de los $\operatorname{casos}^{37}$. Estas intervenciones incluyen CPRE, drenajes percutáneos o realizar colecistectomía completa. La CST laparoscópica requiere de habilidades laparoscópicas avanzadas para un tratamiento exitoso ${ }^{38}$. 


\section{Conclusión}

La colecistectomía es un procedimiento frecuente para el cirujano general, por lo que es fundamental que todo médico residente de cirugía general tenga una formación óptima y especializada para el manejo de los distintos escenarios con los que se presentan las enfermedades de la vesícula biliar, con la finalidad de resolver de forma segura la patología que aqueja a los pacientes.

\section{Cumplimiento de normas éticas}

Consentimiento informado. Los autores declaran que para esta investigación no se han realizado experimentos en seres humanos ni en animales. También declaran que en este artículo no aparecen datos de pacientes, por lo tanto, no se requiere diligenciamiento de consentimiento informado. Se han seguido los protocolos de su centro de trabajo sobre la publicación de datos de pacientes.

Conflicto de intereses: Los autores declaran no tener ningún conflicto de intereses.

Fuentes de financiación: Este estudio se realizó con recursos de los autores.

\section{Contribución de los autores:}

- Concepción y diseño del estudio: Alfredo Chama- Naranjo y Víctor José Cuevas-Osorio.

- Adquisición, análisis e interpretación de datos: Alfredo Chama-Naranjo.

- Redacción del manuscrito: Alfredo Chama-Naranjo y Jorge Farell-Rivas.

- Revisión crítica: Alfredo Chama-Naranjo, Jorge Farell-Rivas y Víctor José Cuevas-Osorio.

\section{Referencias}

1. Everhart JE, Ruhl CE. Burden of digestive diseases in the United States Part III: Liver, biliary tract, and pancreas. Gastroenterology. 2009;136:1134-44. httsp://doi.org/10.1053/j.gastro.2009.02.038

2. Granados-Romero JJ, Cabal-Jiménez KE, Martínez-Carballo G, Nieva-Kehoe R, Londaiz-Gómez R. Colecistectomía laparoscópica en pacientes mayores de 60 años. Experiencia de un hospital de tercer nivel. Cir Cir. 2001;69:271-5.

3. Osuna-Rubio J, Hermosillo-Sandoval JM, Lopéz-Guillén G, Maciel-Miranda A, Fuentes-Orozco C, Álvarez-Villaseñor AS, et al. Efecto del tamaño de la incisión en la gravedad de la lesión de la vía biliar iatrogénica posterior a colecistectomía abierta. Gac Med Mex. 2008;144:213-8.
4. CENETEC. Guía de referencia rápida: Diagnóstico y tratamiento de colecistitis y colelitiasis. Guía de práctica clínica. México; 2011. Fecha de consulta: 02-04-2020. Disponible en: http://www.cenetec.salud.gob.mx/descargas/gpc/CatalogoMaestro/237_IMSS_09_Colecistitis_Colelitiasis/GRR_IMSS_237_09.pdf

5. Majumder A, Altieri MS, Brunt LM, How do I do it: laparoscopic cholecystectomy. Ann Laparosc Endosc Surg. 2020;5:15 httsp://doi.org/10.21037/ales.2020.02.06

6. Novitsky YW, Kercher KW, Czerniach DR, Kaban GK, Khera S, Gallagher-Dorval KA, et al. Advantages of mini-laparoscopic vs conventional laparoscopic cholecystectomy. Results of a prospective randomized trial. Arch Surg. 2005;140:1178-83. httsp://doi.org/10.1001/archsurg.140.12.1178

7. Harris HW. Biliary system. In: Norton J, Barie PS, Bollinger RR, Chang AE, Lowry S, Mulvihill SJ, et al. (eds.). Surgery. Basic science and clinical evidence. $2^{\text {nd }}$ edition. Springer: New York; 2008. P. 911-42.

8. Pucher PH, Brunt LM, Davies N, Linsk A, Munshi A, Rodriguez HA, et al. Outcome trends and safety measures after 30 years of laparoscopic cholecystectomy: a systematic review and pooled data analysis. Surg Endosc. 2018;32:2175-83 https://doi.org/10.1007/s00464-017-5974-2

9. Strasberg SM, Hertl M, Soper NJ. An analysis of the problem of biliary injury during laparoscopic cholecystectomy. J Am Coll Surg. 1995;180:101-25.

10. Kern KA. Malpractice litigation involving laparoscopic cholecystectomy: Cost, cause, and consequences. Arch Surg. 1997;132:392-8.

https://doi.org/10.1001/archsurg.1997.01430280066009

11. Strasberg SM, Brunt LM. Rationale and use of the critical view of safety in laparoscopic cholecystectomy. J Am Col Surg. 2010;211:132-8. https://doi.org/10.1016/j.jamcollsurg.2010.02.053

12. Gómez D, Hernández JD, Caycedo N, Larios GL, Quintero DC. Colecistectomía total en colecistitis complicada: ¿es una conducta segura? Rev Colomb Cir. 2019;34:29-36. https://doi.org/10.30944/20117582.95

13. American College of Surgeons. Strong for surgery. Fecha de consulta: 12-03-2020. Disponible en: https://www.facs.org/quality-programs/strong-for-surgery

14. ASGE Standards of Practice Committee, Buxbaum JL, Fehmi SMA, Sultan S, Fishman DS, Qumseya BJ, Cortessis VK, et al. ASGE guideline on the role of endoscopy in the evaluation and management of choledocholithiasis. Gastrointest Endosc. 2019;89:1075-1105.e15. https://doi.org/10.1016/j.gie.2018.10.001

15. Matsui Y, Satoi S, Kaibori M, Toyokawa H, Yanagimoto H, Matsui K, et al. Antibiotic prophylaxis in laparoscopic cholecystectomy: a randomized controlled trial. PLoS One. 2014;9:e106702. https://doi.org/10.1371/journal.pone.0106702 
16. Lindberg F, Bjorck M, Rasmussen I, Bergqvist D. Current use of thromboembolism prophylaxis for laparoscopic cholecystectomy patients in Sweden. Surg Endosc. 2005;19:386-8. https://doi.org/10.1007/s00464-004-9095-3

17. Mehrotra S, Patnaik PK. Nasogastric decompression in cholecystectomy, is ir necessary? Med J Armed Forces India. 2000;56:17-20. https://doi.org/10.1016/S0377-1237(17)30082-5

18. Hata T, Noda T, Shimizu J, Hatano H, Dono K. Omitting perioperative urinary catheterization in laparoscopic cholecystectomy: a single-institution experience. Surg Today. 2017;47:928-33. https://doi.org/10.1007/s00595-016-1454-x

19. Gupta AK, Shiwach N, Gupta S, Gupta S, Goel A, Bhagat TS. Predicting difficult laparoscopic cholecystectomy. Int Surg J. 2018;5:1094-109. https://doi.org/10.18203/2349-2902.isj20180837

20. Becker OM, Azevedo JL, Azevedo OC, Mendes-Hypólito $\mathrm{OH}$, Miyahira SA, Soares-Miguel GP, et al. Veress needle insertion into the left hypochondrium for creation of pneumoperitoneum: diagnostic value of test to determine the position of the needle in unselected patients. Rev. Col. Bras. Cir. 2011;38:28-34. http://dx.doi.org/10.1590/S0100-69912011000100006

21. Barazanchi AWH, MacFater WS, Rahiri JL, Tutone S, Hill AG, Joshi GP, on behalf of the PROSPECT collaboration. Evidence-based management of pain after laparoscopic cholecystectomy: a PROSPECT review update. Br J Anaesth. 2018:121:787e803. https://doi.org/10.1016/j.bja.2018.06.023

22. Singh M, Prasad N. The anatomy of Rouviere's sulcus as seen during laparoscopic cholecystectomy: A proposed classification. J Minim Access Surg. 2017;13:89-95. https://doi.org/10.4103/0972-9941.201731

23. Thacoor A, Pike TW, Pathak S, Dixon J, Macutkiewicz C, Smith AM. The role of intraoperative cholangiography in patients undergoing laparoscopic cholecystectomy for acute gallstone pancreatitis: is magnetic resonance cholangiopancreatography needed? Ann R Coll Surg Engl. 2019;101:428-31. https://doi.org/10.1308/rcsann.2019.0049

24. La Regina D, Mongelli F, Cafarotti S, Saporito A, Ceppi M, Di Giuseppe M, et al. Use of retrieval bag in the prevention of wound infection in elective laparoscopic cholecystectomy: is it evidence-based? A meta- analysis. BMC Surg. 2018;18:102. https://doi.org/10.1186/s12893-018-0442-z

25. Bunting DM. Port-site hernia following laparoscopic cholecystectomy. JSLS. 2010;14:490-7. https://doi.org/10.4293/108680810X12924466007728

26. Gan TJ, Meyer T, Apfel CC, Chung F, Davis PJ, Habib AS, et al. Society for ambulatory anesthesia guidelines for the management of postoperative nausea and vomiting. Anesth Analg. 2007;105:1615-28.
27. Siada SS, Schaetzel SS, Chen AK, Hoang HD, Wilder FG, Dirks RC, et al. Day versus night laparoscopic cholecystectomy for acute cholecystitis: a comparison of outcomes and cost. Am J Surg. 2017;214:1024-7. https://doi.org/10.1016/j.amjsurg.2017.08.027

28. Bisgaard T, Warltier DC. Analgesic treatment after laparoscopic cholecystectomy: a critical assessment of the evidence. Anesthesiology. 2006;104:835-46. https://doi.org/10.1097/00000542-200604000-00030

29. Cano-Zepeda NI, De Gante-Aguilar JM. Cultura de seguridad, estrategia para prevenir la disrupción de la vía biliar. Cir Gen. 2018;40:179-83.

30. Keus F, de Jong JAF, Gooszen HG, Van Laarhoven CJ. Laparoscopic versus open cholecystectomy for patients with symptomatic cholecystolithiasis. Cochrane Database Syst Rev. 2006:CD006231. https://doi.org/10.1002/14651858.CD006231

31. Buddingh KT, Weersma RK, Savenije RAJ, van Dam GM, Nieuwenhuijs VB. Lower rate of major bile duct injury and increased intraoperative management of common bile duct stones after implementation of routine intraoperative cholangiography. J Am Coll Surg. 2011;213:267-74. https://doi.org/10.1016/j.jamcollsurg.2011.03.004

32. Traverso LW. Intraoperative cholangiography lowers the risk of bile duct injury during cholecystectomy. Surg Endosc. 2006;20:1659-61. https://doi.org/10.1007/s00464-006-0122-4

33. Alvarez LF, Rivera D, Esmeral ME, García MC, Toro DF, Rojas OL. Colecistectomía laparoscópica difícil, estrategias de manejo. Rev Colomb Cir. 2013;28:186-95.

34. Strasberg SM. A three-step conceptual roadmap for avoiding bile duct injury in laparoscopic cholecystectomy: an invited perspective review. J Hepatobiliary Pancreat Sci. 2019;26:123-7. https://doi.org/10.1002/jhbp.616

35. Estes WL. Acute gangrenous cholecystitis and the use of partial cholecystectomy in its treatment. Am J Surg. 1939;40:197-204.

36. Shin M, Choi N, Yoo Y, Kim Y, Kim S, Mun S. Clinical outcomes of subtotal cholecystectomy performed for difficult cholecystectomy. Ann Surg Treat Res. 2016;91:226-32. https://doi.org/10.4174/astr.2016.91.5.226

37. Lidsky ME, Speicher PJ, Ezekian B, Holt EW, Nussbaum DP, Castleberry AW, et al. Subtotal cholecystectomy for the hostile gallbladder: failure to control the cystic duct results in significant morbidity. HPB. 2017;19:547-56. https://doi.org/10.1016/j.hpb.2017.02.441

38. Strasberg SM, Pucci MJ, Brunt LM, Deziel DJ. Subtotal cholecystectomy-"fenestrating" vs "reconstituting" subtypes and the prevention of bile duct injury: definition of the optimal procedure in difficult operative conditions. J Am Coll Surg. 2016;222:89-96. https://doi.org/10.1016/j.jamcollsurg.2015.09.019 